DOI: 10.17516/1997-1397-2021-14-5-611-623

УДК 537.62

\title{
Features in the Resonance Behavior of Magnetization in Arrays of Triangular and Square Nanodots
}

\author{
Vitaly A. Orlov* \\ Siberian Federal University \\ Krasnoyarsk, Russian Federation \\ Kirensky Institute of Physics \\ Federal Research Center KSC SB RAS \\ Krasnoyarsk, Russian Federation \\ Roman Yu. Rudenko ${ }^{\dagger}$ \\ Siberian Federal University \\ Krasnoyarsk, Russian Federation \\ Vladimir S. Prokopenko ${ }^{\ddagger}$ \\ Irina N. Orlova ${ }^{\S}$
}

Krasnoyarsk State Pedagogical University named after V. P. Astafiev

Krasnoyarsk, Russian Federation

Received 12.04.2021, received in revised form 15.06.2021, accepted 25.06.2021

\begin{abstract}
Collective modes of the gyrotropic motion of a magnetic vortex core in ordered arrays of triangular and square ferromagnetic film nanodots have been theoretically investigated. The dispersion relations have been derived. The dipole-dipole interaction of the magnetic moments of the magnetic vortex cores of elements has been taken into account in the approximation of a small shift from the equilibrium position. It is shown that the effective rigidity of the magnetic subsystem of triangular elements is noticeably higher than that of the subsystem of square elements of the same linear sizes. The potential application of the polygonal film nanodisks as nanoscalpels for noninvasive tumor cell surgery is discussed.
\end{abstract}

Keywords: differential equation, Cauchy problem, split, stability, convergence.

Citation: V.A. Orlov, R.Yu. Rudenko, V.S. Prokopenko, I.N. Orlova, Features in the Resonance Behavior of Magnetization in Arrays of Triangular and Square Nanodots, J. Sib. Fed. Univ. Math. Phys., 2021, 14(5), 611-623. DOI: 10.17516/1997-1397-2021-14-5-611-623.

\section{Introduction}

Ordered arrays and suspensions of ferromagnetic nanodots have a great potential for application in new spintronic devices and noninvasive cell nanosurgery of malignant tumors in medicine $[1,2]$. The requirements for the magnetic moment of a nanoobject used as a magneticfiled-driven nanoscalpel for cell destruction are contradictory: an increase in the magnetic moment facilitates the cell destruction, but is accompanied by an undesirable effect of agglomeration

\footnotetext{
*vaorlov@sfu-kras.ru https://orcid.org/0000-0002-9025-0075

${ }^{\dagger}$ rudenko-roma406@yandex.ru https://orcid.org/0000-0002-2488-3624

${ }^{\ddagger}$ plufe@yandex.ru

§bondhome@mail.ru https://orcid.org/0000-0002-8638-3879

(c) Siberian Federal University. All rights reserved
} 
of nanoparticles in a suspension. To resolve this contradiction, it is necessary to search for a compromise situation where the initial magnetic moment of a nanoscalpel is small, but significantly increases upon switching on a magnetic field at the instant of destruction.

The aim of this work, along with studying the features in the resonance behavior of the magnetization of ordered arrays of triangular and square nanodots, is to clarify whether the problem of nanoparticle agglomeration in a cell surgery suspension can be solved by changing the nanoparticle geometry.

The point is that the disk is not an optimal nanoscalpel configuration. In ferromagnetic nanoparticles suspended in a liquid, the magnetic flux tends to close inside an element with the formation of magnetic vortices. In a disk-shaped particle, a single vortex is formed (Fig. 1a), in which the magnetization is circularly oriented in the plane at the periphery of a particle and is out-of-plane at its center (the vortex core). The magnetic moment of an object is induced mainly by the core. The external field causes a reversible displacement of the core at the almost invariable value of the magnetic moment.

For the analysis, we chose square and triangle configurations as presumably promising. As in disks, the magnetic flux in square and triangular nanodots is almost completely closed within an element. A quasi-vortex with a core at the center is formed (Fig. $1 \mathrm{~b}$ and c). A core is the magnet region with a size of $\delta_{0} \approx 10 \mathrm{~nm}$ in which the magnetization is out-of-plane (perpendicular to the magnet plane) due to the competition between the exchange and demagnetizing energies: $\delta_{0} \approx \sqrt{A /\left(\mu_{0} M_{S}\right)}\left(A\right.$ is the exchange constant and $M_{S}$ is the saturation magnetization).

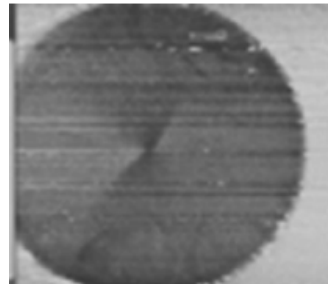

a)

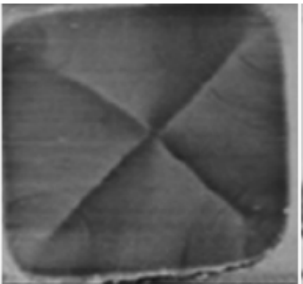

b)

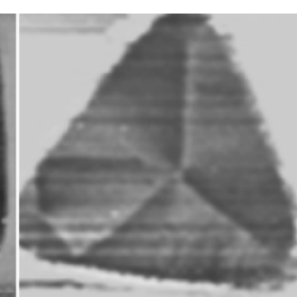

c)

Fig. 1. Equilibrium magnetic structures of (a) circular, (b) square, and (c) triangular permalloy film nanospots [3]. The quasi-vortices of the square and triangular spots represent closed domain structures with a vortex core at the center

Under the action of any factors (external fields, spin-polarized currents, anisotropy field gradients, stresses, etc.), a magnetic vortex moves along a curvilinear trajectory, being driven by the Magnus forces [4-8]. In analytical calculations, the well-proven rigid vortex approximation is often used. In the model used, it is assumed that the magnetization configuration in the region covered by the vortex distribution remains unchanged upon displacement of the core from the equilibrium position. In this case, the vortex dynamics should be described by the method of collective variables, which are the core coordinate and velocity. Then, the equations of motion take the well-known form (the Thiele equation [9]):

$$
\mathbf{G} \times \mathbf{v}+\widehat{D} \mathbf{v}+\nabla W=0 .
$$

Here $\mathbf{G}$ is the gyrovector $[7,9,10]$, perpendicular to the magnet plane, $\mathbf{v}$ is the core velocity, $W$ is the potential energy of the vortex, and $\widehat{D}$ is the tensor of effective coefficients of the force of friction. The potential energy $W$ includes the terms responsible for its growth due to the 
exit of the magnetic subsystem of elements from the metastable state (the shift of the core from the equilibrium position), the terms describing the pairing energy of interaction between the magnetic moments of different elements, and the terms describing external factors (fields).

Below, we consider specific equations for square and triangular elements using the models that are simple, but make it possible to compare the resonance behavior of disks with different shapes and the effective rigidity of their magnetic subsystems.

\section{Effective Potential Energy of a Polygonal Ferromagnetic Element}

In this Section, we estimate the increment of the potential energy $W$ of magnetic elements upon displacement of the magnetic vortex core from the equilibrium position. A rigorous analytical solution to this problem faces great computational difficulties. Therefore, the numerical modeling is frequently used (see, for example, [11-14]). This calculation is necessary to determine the parameters of vortex motion using Eq. (1).

Fig. 2. presents the models of ferromagnetic elements. Each element has a domain magnetization structure with a vortex at the center of a magnet. To perform the estimation, we assume that, upon displacement of the core, the energy of the magnetic subsystem changes mainly due to an increase in the energy of the magnetostatic interaction of domains. We calculate this energy using the dipole-dipole approximation. It can be shown that the energy of the magnetostatic interaction of uniformly magnetized triangular regions (domains) can be approximately presented as the energy of interaction between dipoles located in the medians at a distance of one third of the height from the base to which the magnetization is parallel (quasi-dipoles). In Fig. 2, these positions are shown by closed circles with arrows. The value of magnetic moment $\mathbf{M}$ of each dipole is determined by the magnetic moment of the corresponding magnetic domain.

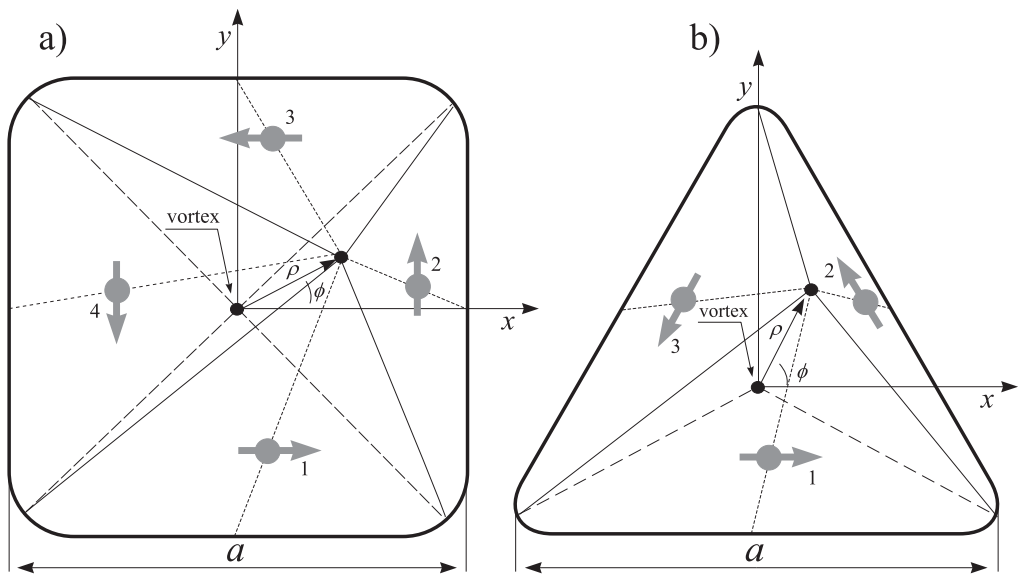

Fig. 2. Models of ferromagnetic nanoelements in the form of a square and a regular triangle

When the core is shifted from the element centers, the domain structure configuration changes, which is reflected in a change in the domain size and, consequently, in the energy of the interaction between domains. This process can be considered as a variation in the energy of interaction between magnetic dipoles (quasi-dipoles), which, in this case, shifted and changed the absolute values of their magnetic moments. 
Let us enumerate domains as shown in Fig. 2. For the energy of interaction between the $n$-th and $m$-th dipoles, we write

$$
W_{n m}=\frac{\mu_{0}}{4 \pi}\left(\frac{\mathbf{M}_{n} \mathbf{M}_{m}}{r_{n m}^{3}}-3 \frac{\left(\mathbf{M}_{n} \mathbf{r}_{n m}\right)\left(\mathbf{M}_{m} \mathbf{r}_{n m}\right)}{r_{n m}^{5}}\right) .
$$

Here $\mathbf{r}_{n m}$ is the radius vector connecting the dipoles and $\mathbf{M}_{n}$ and $\mathbf{M}_{m}$ are their magnetic moments. For the magnetic moments, we can write

$$
\left|\mathbf{M}_{n}\right| \approx M_{S} V_{n}=\frac{1}{2} M_{S} b S_{n}=\frac{1}{2} M_{S} b a h_{n} .
$$

Here $M_{S}$ is the saturation magnetization, $V_{n}$ is the domain (prism) volume, $S_{n}$ is the domain square (triangle), $h_{n}$ is the altitude of a triangular domain plotted from the core position to the outer side of the element, $b$ - is the element thickness, and $a$ is the side of a regular polygon.

The vortex core position is specified by length $\rho$ of its radius vector and azimuth angle $\phi$. Obviously, the dipole positions and absolute values of the magnetic moments are determined by the $\rho$ and $\phi$ values. Solving a simple geometric problem, we can express the parameters included in energy (2) through $\rho$ and $\phi$. The results of the calculation are given in Tab. 1.

Table 1. Parameters of quasi-dipoles as functions of $\rho$ and $\phi$ according to the numeration in Fig. 2. The absolute values of the magnetic moments are calculated using Eq. (3)

\begin{tabular}{|c|l|l|}
\hline $\begin{array}{c}\text { Element } \\
\text { shape }\end{array}$ & \multicolumn{1}{|c|}{$\begin{array}{c}\text { Quasi-dipoles Cartesian } \\
\text { coordinates }\end{array}$} & \multicolumn{1}{c|}{ Quasi-dipole magnetic moment } \\
\hline \multirow{5}{*}{ Square } & $x_{1}=\frac{1}{3} \rho \cos (\phi)$ & \\
$x_{2}=\frac{1}{3}(a+\rho \cos (\phi))$ & $M_{1}=\frac{1}{2} M_{S} b a\left(\frac{a}{2}+\rho \sin (\phi)\right)$ \\
$x_{3}=x_{1}$ & $M_{2}=\frac{1}{2} M_{S} b a\left(\frac{a}{2}-\rho \cos (\phi)\right)$ \\
& $x_{4}=-\frac{1}{3}(a-\rho \cos (\phi))$ & $M_{3}=\frac{1}{2} M_{S} b a\left(\frac{a}{2}-\rho \sin (\phi)\right)$ \\
& $y_{1}=-\frac{1}{3}(a-\rho \sin (\phi))$ & $M_{4}=\frac{1}{2} M_{S} b a\left(\frac{a}{2}+\rho \cos (\phi)\right)$ \\
\hline \multirow{5}{*}{ Triangle } & $\begin{array}{l}y_{3}=\frac{1}{3}(a+\rho \sin (\phi) \\
y_{4}=y_{2} .\end{array}$ & $M_{1}=\frac{1}{2} M_{S} b a\left(\frac{a \sqrt{3}}{6}+\rho \sin (\phi)\right)$ \\
& $x_{1}=\frac{1}{3} \rho \cos (\phi)$ & $M_{2}=\frac{1}{4} M_{S} b a\left(\frac{a \sqrt{3}}{3}-\sqrt{3} \rho \cos (\phi)-\rho \sin (\phi)\right)$ \\
$x_{2}=\frac{1}{3}\left(\frac{a}{2}+\rho \cos (\phi)\right)$ & $M_{3}=\frac{1}{4} M_{S} b a\left(\frac{a \sqrt{3}}{3}+\sqrt{3} \rho \cos (\phi)-\rho \sin (\phi)\right)$ \\
& $x_{3}=-\frac{1}{3}\left(\frac{a \sqrt{3}}{3}-\rho \cos (\phi)\right)$ & \\
\hline & $y_{2}=\frac{1}{3}\left(\frac{a \sqrt{3}}{6}+\rho \sin (\phi)\right)$ \\
$y_{3}=y_{2}$ & \\
\hline
\end{tabular}

Taking into account the data from Tab. 1, we obtain the square element energy

$$
W_{s q}(\rho)=W_{12}+W_{13}+W_{14}+W_{23}+W_{24}+W_{34}=\frac{27}{128} \frac{\mu_{0} M_{S}^{2} b^{2}}{\pi a}\left(\rho^{2}-\frac{a^{2}}{2}\right),
$$


and the triangular element energy

$$
W_{t r}(\rho)=W_{12}+W_{13}+W_{32}=\frac{15}{2} \frac{27}{128} \frac{\mu_{0} M_{S}^{2} b^{2}}{\pi a}\left(\rho^{2}-\frac{a^{2}}{3}\right) .
$$

Importantly, according to the calculation, the energies of both a square and triangular element are independent of the azimuth angle; i.e. the potential of the vortex core has a cylindrical symmetry. This is a safety signal, since the potential does not reflect the shape of a magnet. This is true until the area covered by the vortex reaches the boundary of a magnet. If the shift is so large that the core appears in the vicinity of the boundary $(\rho \approx a)$, then the vortex distribution will lead to the occurrence of noticeable magnetostatic charges on the lateral surface. The contribution of the terms related to these charges to the magnetostatic energy will ensure the dependence of the total potential energy on the lateral surface shape. In this case, the symmetry of a magnet will be reflected in the functional dependence of the energy on azimuth angle $\phi$ [15-18]. Thus, Eqs. (4) and (5) are valid as long as the core shift does not lead to the exit of the magnetization from the lateral surface of a magnet.

The analytical form of Eqs. (4)-(5) allows us to estimate the initial susceptibilities of nanoelements. To do that, we obtain the dependence of the total magnetic moment of an element on applied dc magnetic field $H$. For simplicity, we assume that the magnetic field is parallel to the polygon side (directed along the $O X$ axis for both the square and the triangle).

When the external field is applied, the energy of the magnetic subsystem should be added with the Zeeman energy

$$
W_{t o t}=W(\rho)-\mathbf{M H} .
$$

Switching on the field $\mathbf{H}$, along the positive direction of the $x$ axis (see Fig. 2), leads to the displacement of the core from the magnet center in the positive direction of the $y$ axis $(\phi=\pi / 2$, $q=+1)$. or in the opposite direction $(\phi=-\pi / 2)$ if the magnetization in elements rotates clockwise $(q=-1)$ rather than counterclockwise.

The total magnetic moment of an element can be calculated as follows (see Fig. 3). A domain with the magnetization co-directed with the field grows by the expense of domains the magnetization of which has the energetically unfavorable direction. Then, for the magnetic moment of a square element, obtain

$$
|\mathbf{M}|=\frac{1}{2} M_{S} b a\left(\frac{a}{2}+\rho\right)-\frac{1}{2} M_{S} b a\left(\frac{a}{2}-\rho\right)=M_{S} b a \rho .
$$

The search for the equilibrium shift $\rho$ is conventionally made with regard to Eq. (7):

$$
\frac{d W_{t o t}}{d \rho}=\frac{54}{128} \frac{\mu_{0} M_{S}^{2} b^{2}}{\pi a} \rho-M_{S} b a H=0 .
$$

Then, for the equilibrium position of the core, obtain

$$
\rho=\frac{128}{54} \frac{\pi a^{2}}{\mu_{0} M_{S} b} H .
$$

Taking into account (9), for magnetic moment (7) obtain

$$
M=\chi_{s q} H, \quad \chi_{s q}=\frac{128}{54} \frac{\pi a^{3}}{\mu_{0}} .
$$

The calculation for triangular elements, analogously to (7)-(10) yields

$$
\chi_{t r}=\frac{24}{135} \frac{\pi a^{3}}{\mu_{0}} .
$$


a)

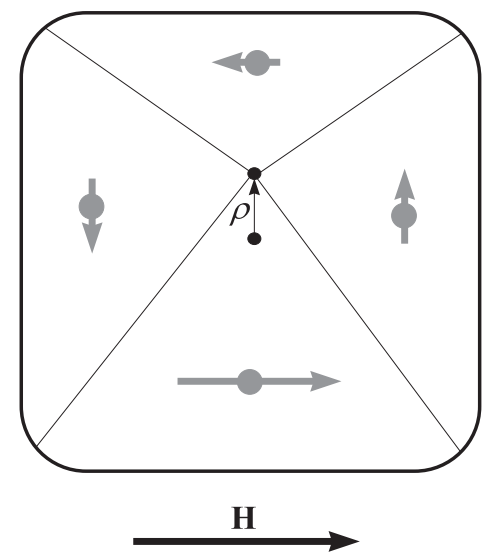

b)

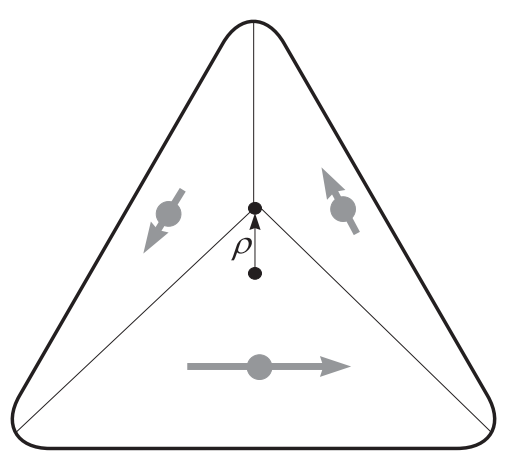

Fig. 3. Occurrence of the magnetic moment in (a) a square and (b) triangular element

\section{Collective Modes in an Ordered Array of Polygons}

The above analytical expressions for energy $W(\rho)$ allow us to analyze the gyrotropic motion of vortex cores in the arrays of elements arranged in a certain order, for example, forming a square lattice (see Fig. 4 for triangular elements).

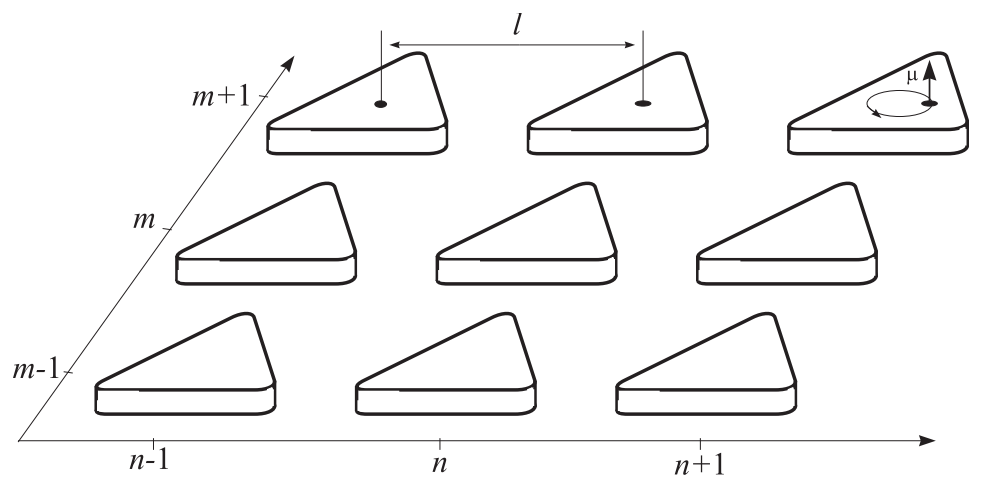

Fig. 4. Example of an ordered array of triangular elements. The distance between the centers of elements is $l$. Example of orientation of the magnetization of the core and trajectory of its motion

Let us continue the discussion of the case when the vortex cores in elements remain fairly distant from the element edges during the motion induced by, e.g., an ac magnetic field. In this case, the magnetic subsystems of triangles interact magnetostatically only due to the presence of a magnetic moment of cores at the center of the vortices. At the vortex center, the magnetization is perpendicular to the element surface and, depending on the $p$ polarity, can be conditionally oriented upward $(p=1)$ or downward $(p=-1)$. The example of the trajectory of the core motion in the centrosymmetric potential is shown in Fig. 4 for one of the elements. The direction of the gyrotropic rotation is determined by the sign of the product of polarity and chirality: $q= \pm 1$.

The energy of interaction of the magnetic moments of the cores of all elements must be included in the total energy of a system. The energy of the pairwise interaction of the core of 
some selected element with polarity $p$ and another element with polarity $p_{n m}$, which belongs to the column with number $n$ in the row with number $m$ can be presented in the form

$$
W_{n m}^{(d i p)}=p p_{n m} \frac{\mu_{0}}{4 \pi} \frac{\mu^{2}}{r_{n m}^{3}} .
$$

Here $r_{n m}=\sqrt{\left(n l+\left(x_{n m}-x\right)\right)^{2}+\left(m l+\left(y_{n m}-y\right)\right)^{2}}$ is the distance between the cores, $\mu$ is the magnetic moment of the core vortex, $x$ and $y$ are the core shifts along the $x$ and $y$ axes of the chosen element, respectively, and $x_{n m}$ and $y_{n m}$ are the core shifts along the $x$ and $y$ axes of the second element with coordinates $(n, m)$, respectively. The summation is made over all $n$ and $m$ except for the case $n=m=0$. For the total energy of the interaction between the chosen element and the rest matrix, we write

$$
W^{(d i p)}=\frac{p \mu_{0} \mu^{2}}{4 \pi} \sum_{n, m} \frac{p_{n m}}{r_{n m}^{3}} .
$$

Then, taking into account (4) or (5), the force acting on the core is

$$
\begin{aligned}
\mathbf{f}=-\nabla\left(W(\rho)+W^{(d i p)}\right) & =\mathbf{e}_{x}\left(-\kappa x+\xi p \sum_{n, m} \frac{p_{n m}\left(4 n^{2}-m^{2}\right)\left(x-x_{n m}\right)}{\left(n^{2}+m^{2}\right)^{\frac{7}{2}}}\right)+ \\
& +\mathbf{e}_{y}\left(-\kappa y+\xi p \sum_{n, m} \frac{p_{n m}\left(4 m^{2}-n^{2}\right)\left(y-y_{n m}\right)}{\left(n^{2}+m^{2}\right)^{\frac{7}{2}}}\right) .
\end{aligned}
$$

The designations used here are $\kappa=54 \mu_{0} M_{S}^{2} b^{2} /(128 \pi a)$ for squares or $\kappa=405 \mu_{0} M_{S}^{2} b^{2} /(128 \pi a)$ for triangles; $\mathbf{e}_{x}$ and $\mathbf{e}_{y}$ are the unit vectors of the $x$ and $y$ axes, respectively; and $\xi=$ $3 \mu_{0} \mu^{2} /\left(4 \pi l^{5}\right)$. In addition, the relations $\rho^{2}=x^{2}+y^{2}, x, y, x_{n m}, y_{n m} \ll l$. were used. It should be noted that Eqs. (14) are valid at the symmetric distribution of the polarities of elements in an infinite array. For instance, at $p_{n m}= \pm 1$, regardless of $n$ and $m$, we have either $p_{n m}=(-1)^{n+m}$ or $p_{n m}=(-1)^{n}$, etc., since, in these cases, the core of a selected element is not affected by constant forces from the side of its neighbors and has an equilibrium position at the center of this element.

According to Eq. (14), we write equation of motion (1) in the components

$$
\left\{\begin{array}{l}
G v_{y}+D v_{x}+\left(\kappa+\xi \sum_{n, m} \frac{p p_{n m}\left(4 n^{2}-m^{2}\right)}{\left(n^{2}+m^{2}\right)^{\frac{7}{2}}}\right) x-\xi \sum_{n m} \frac{p p_{n m}\left(4 n^{2}-m^{2}\right) x_{n m}}{\left(n^{2}+m^{2}\right)^{\frac{7}{2}}}=0, \\
-G v_{x}+D v_{y}+\left(\kappa+\xi \sum_{n, m} \frac{p p_{n m}\left(4 m^{2}-n^{2}\right)}{\left(n^{2}+m^{2}\right)^{\frac{7}{2}}}\right) y-\xi \sum_{n m} \frac{p p_{n m}\left(4 m^{2}-n^{2}\right) y_{n m}}{\left(n^{2}+m^{2}\right)^{\frac{7}{2}}}=0 .
\end{array}\right.
$$

It is reasonable to search for solutions of system of equations (15) in the form of waves

$$
x_{n m}(\mathbf{r}, t)=X e^{i(\mathbf{k r}-\Omega t)}, \quad y_{n m}(\mathbf{r}, t)=i Y e^{i(\mathbf{k r}-\Omega t)} .
$$

Here $i=\sqrt{-1}$ is the imaginary unit, $\mathbf{k}=k_{x} \mathbf{e}_{x}+k_{y} \mathbf{e}_{y}$ is the wave vector, $\mathbf{r}=n l \mathbf{e}_{x}+m l \mathbf{e}_{y}$ is the radius vector connecting the centers of the chosen element and the element for the column with number $n$ and the row with number $m$, and $X$ and $Y$ are the amplitudes of the core shift along the $x$ and $y$ axes, respectively.

Substituting the trial solutions of (16) into system of equations (15), we obtain the system of algebraic equations of variables $X$ and $Y$

$$
\left\{\begin{array}{l}
G \Omega Y-\left(i D \Omega-\kappa-\xi S_{x}\left(k_{x}, k_{y}\right)\right) X=0 \\
G \Omega X-\left(i D \Omega-\kappa-\xi S_{y}\left(k_{x}, k_{y}\right)\right) Y=0
\end{array}\right.
$$


Here, we used the designations

$$
\begin{aligned}
& S_{x}\left(k_{x}, k_{y}\right)=\sum_{n m} \frac{4 n^{2}-m^{2}}{\left(n^{2}+m^{2}\right)^{\frac{7}{2}}}\left(1-\cos \left(k_{x} n l\right) \cos \left(k_{y} m l\right)\right), \\
& S_{y}\left(k_{x}, k_{y}\right)=\sum_{n m} \frac{4 m^{2}-n^{2}}{\left(n^{2}+m^{2}\right)^{\frac{7}{2}}}\left(1-\cos \left(k_{x} n l\right) \cos \left(k_{y} m l\right)\right) .
\end{aligned}
$$

Equating the determinant built on the coefficients at $X$ and $Y$ to zero, we obtain the quadratic equation of variable $\Omega$, the solution of which is

$$
\Omega= \pm \omega-i \delta
$$

where

$$
\begin{gathered}
\omega=\left[\frac{\left(\kappa+\xi S_{x}\left(k_{x}, k_{y}\right)\right)\left(\kappa+\xi S_{y}\left(k_{x}, k_{y}\right)\right)}{G^{2}+D^{2}}-\frac{\kappa^{2} D^{2}}{\left(G^{2}+D^{2}\right)^{2}}\right]^{\frac{1}{2}}, \\
\delta=\frac{\kappa D}{G^{2}+D^{2}} .
\end{gathered}
$$

The real part of Eq. (19) - $\omega$ - determines the angular velocity of the vortex core rotation (the gyrotropic frequency). The imaginary part $\delta$ determines the effective damping parameter.

In the long-wavelength limit $\left(k_{x}, k_{y} \rightarrow 0\right)$, the sums turn to zero and we obtain the well-known expressions for the frequency and damping parameter in a single element $[19,20]$. Indeed, in this case, the cores of all disks of the array move synchronously and the dipole-dipole interaction forces acting on a selected element are compensated pairwise from neighbors with numbers $n$ and $-n$, similar to $m$ and $-m$. In other words, the interaction between the elements in this case does not affect the gyrotropic frequency. In other cases, Eq. (20) specifies the dispersion law $\omega\left(k_{x}, k_{y}\right)$.

When creating ordered arrays of ferromagnetic elements, nature chooses the energetically most favorable state, which corresponds to a checkboard pattern of the polarity signs, i.e., the state given by the expression $p_{n m}=(-1)^{n+m}$. In the opposite limit (the least favorable distribution), the polarities of all elements have the same sign (the magnetization at the center of all vortices is identically directed); i.e., $p$ is independent of numbers $n$ and $m$. Such a state can be created by applying a magnetic field exceeding the saturation field perpendicular to the array plane. The dispersion surfaces for these extreme cases are shown in Fig. 5. The linear sizes of the cores are, as a rule, smaller than the sizes of elements; therefore, the inequality $\xi \ll \kappa$ can be considered valid.

Basing on Fig. 5, we should emphasize an important feature. At sufficiently large $\xi$ values, in the model with the alternating $p$ signs, there are the wavenumber ranges in which $\omega$ becomes imaginary, which is indicative of the aperiodic motion of the cores. This can be qualitatively explained as follows. The alternation of the polarity sign leads to a decrease in the effective rigidity of the potential in which the core moves. This obviously follows from Eqs. (15) or (20), where the rigidity $\kappa$ is added with the correction the value and sign of which depend on the values of polarities $p_{n m}$. At different polarity signs, the attraction between the cores of neighboring elements arises (on the contrary to the repulsion at the same $p$ ), which competes with the restoring force determined by the rigidity $\kappa$. of the magnetic subsystem. As a result, the restoring force acting on the core becomes so small that it leads to the imaginary $\omega$ value (20). 

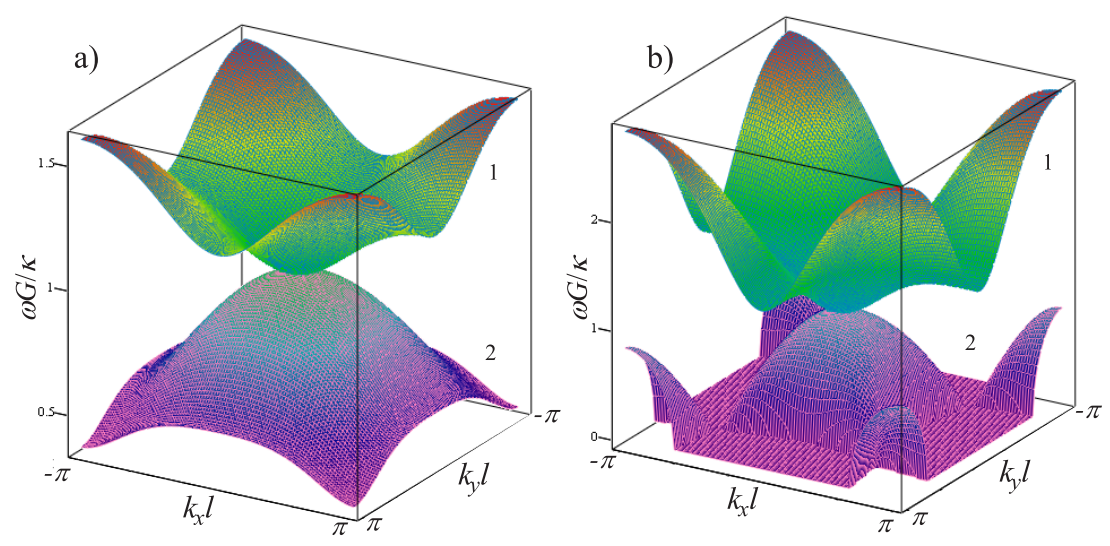

Fig. 5. Dispersion laws $\omega\left(k_{x}, k_{y}\right)$ based on Eq. (20). The frequency in units of $\kappa / G$ is laid off along the vertical axis. (a) The case of $\delta=0.1 \omega$ and $\xi=0.1 \kappa$ and (b) the case of $\xi=0.3 \kappa$. The surfaces with number 1 correspond to the case of the same polarity of vortices of all elements and the surfaces with number 2 , to the case of a checkboard pattern of the polarity signs

\section{Discussion}

To sum up the comparison of the properties of arrays of square and triangular elements, we can emphasize some circumstances that can play a significant role in selecting the objects that are candidates for use as functional tools for medicine or various spintronic devices. First of all, it should be noted that the effective rigidities of the magnetic subsystems of square and triangular elements of the same linear sizes are significantly different. This feature is responsible for the difference between the initial susceptibilities of the arrays of these elements by almost an order of magnitude. According to Eqs. (10) and (11), we have

$$
\frac{\chi_{s q}}{\chi_{t r}}=\frac{128}{54} \frac{135}{24} \approx 13
$$

This phenomenon was confirmed in our experiment. The arrays of square, triangular, and circular elements for the experiment were formed by explosive lithography from a continuous film using high-vacuum thermal sputtering of an $80 \mathrm{HXC}$ alloy onto a silicon substrate coated with a photoresist. The required morphology was formed on the substrate surface using an AZ Nlof 2035 negative photoresist. The magnetic structure and morphology of the obtained elements were examined on a Veeco MultiMode NanoScope IIIa scanning probe microscope.

The surface morphology of the investigated arrays is shown in Fig. 6. The images were obtained on an atomic force microscope operating in a tapping mode [21,22]. A cantilever was brought to the surface so that in the lower half-period of the oscillations, the sample surface was touched. The interaction of the cantilever with the surface in the tapping mode was ensured by the van der Waals forces, which, at the instant of touching, are added with the elastic force acting on the cantilever from the surface side.

Fig. 7 shows a typical scan of the magnetic structure obtained using a two-pass technique in the cantilever frequency modulation mode. The return passage height is $z_{0}=50 \mathrm{~nm}$. The obtained images allow us to conclude that, in square elements, an equilibrium structure with the 

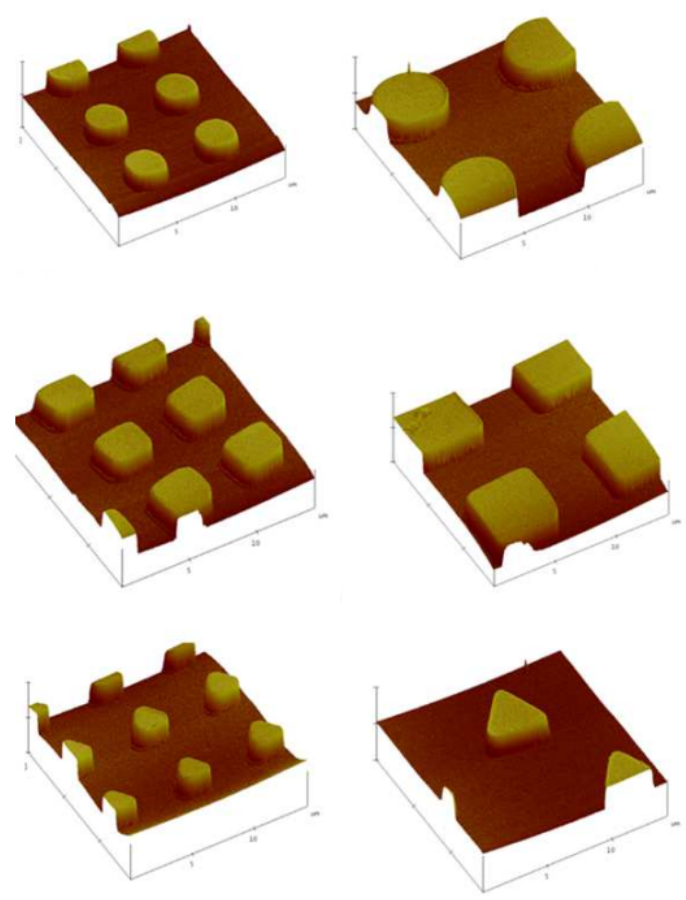

Fig. 6. Atomic force microscopy images of the surface relief of the arrays of different elements. The linear sizes of the elements are the same (the diameter is 3 micrometers and the thickness is $12 \mathrm{~nm}$ )

closed magnetic flux (quasi-vortex) of four domains separated by 90-degree Neel walls is most often implemented, while in triangular elements, the structure is formed by three domains with 120-degree walls. At the center, at the intersection of the diagonals, there is a core similar to that at the center of circular elements.
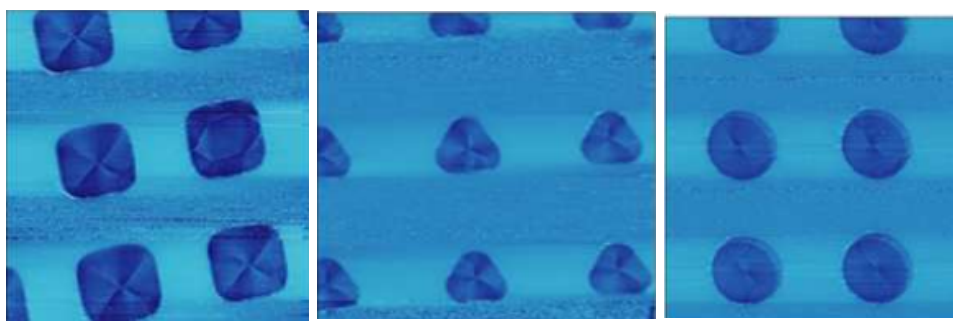

Fig. 7. Atomic force microscopy images of the magnetic structure of different elements

The hysteresis loops of the arrays of squares and triangles were obtained on a NanoMOKE facility. The result is presented in Fig. 8. It can be seen that the slopes of the initial portions of the curves for square and triangular elements differ by more than an order of magnitude, which is in good agreement with estimate (22).

Such a significant dependence of the effective rigidity of the magnetic subsystems on the element shape should be taken into account in designing the basic components, for example, 
storage devices, field sensors, etc. For medicine, square-shaped elements seem to be preferable, since they more readily respond to an external field, which simplifies the control of their motion in suspensions and biological liquids. In zero external field, they have a weak total magnetic moment, since the flux is closed inside an element and the magnetic moment of the central part of the vortex is small due to the small size of the core.
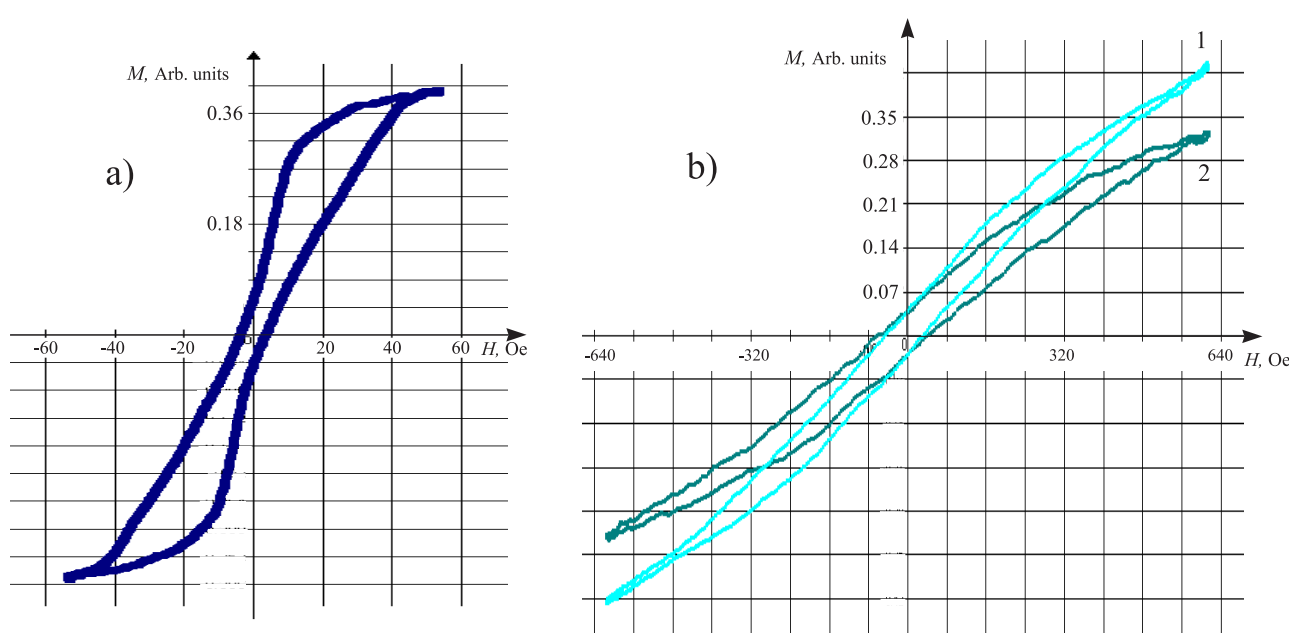

Fig. 8. Hysteresis loops obtained on an array of (a) square and (b) triangular elements. The loop in (a) was obtained by applying a field along the square side. Plot 1 in (b) was obtained by applying a field along the triangle edge and plot 2, by applying a field along the triangle height

In addition, a significant difference in the rigidity of the system will affect the resonance properties of the arrays. The frequencies of the gyrotropic motion of arrays of square elements were about $400 \mathrm{MHz} 400 \mathrm{M \Gamma ц} \mathrm{[15],} \mathrm{while} \mathrm{for} \mathrm{triangular} \mathrm{elements} \mathrm{one} \mathrm{should} \mathrm{expect} \mathrm{multiply}$ higher frequencies. Features of the collective modes for different shapes of elements will be objects of further investigations.

As intermediate results, in this work, the analytical expressions for the potential energy of the magnetic vortex core and the dispersion relations were obtained.

This study was supported by the Russian Foundation for Basic Research, project no. 20-0200696 .

\section{References}

[1] E.A.Vitol, V.Novosad, E.A.Rozhkova, Microfabricated magnetic structures for future medicine: from sensors to cell actuators, Nanomedicine, $\mathbf{7}(2012)$, no. 6, 1611.

[2] G.S.Zamay, O.S.Kolovskaya et al., Aptamers Selected to Postoperative Lung Adeocarcinoma Detect Circulating Tumor Cells in Human Blood, Molecular Therapy, 23(2015), no. 9, 1486-1496. DOI: $10.1038 / \mathrm{mt} .2015 .108$

[3] P.D.Kim, V.S.Prokopenko et al., Magnetic Structures of Permalloy Film Microspots, Doklady Physics, 60(2015), no. 7, 279-282. DOI:10.1134/S1028335815070046 
[4] A.K.Zvezdin, V.I.Belotelov, K.A.Zvezdin, Gyroscopic Force Acting on the Magnetic Vortex in a Weak Ferromagnet, JETP Letters, 87(2008), no. 7, 381-384.

DOI: $10.1134 / \mathrm{S} 0021364008070102$

[5] A.E.Ekomasov, S.V.Stepanov, K.A.Zvezdin, E.G.Ekomasov, Spin current induced dynamics and polarity switching of coupled magnetic vertices in three-layer nanopillars, Journal of Magnetism and Magnetic Materials, 471(2019), 513-52.

[6] B.A.Ivanova, G.G.Avanesyan, A.V.Khvalkovskiy, N.E.Kulagin, C.E.Zaspel, K.A.Zvezdin, NonNewtonian Dynamics of the Fast Motion of a Magnetic Vortex, JETP Letters, 91(2010), no. $4,178-182$. DOI: $10.1134 /$ S0021364010040041

[7] K.Yu.Guslienko, B.A.Ivanov, V.Novosad, Y.Otani, H.Shima K.Fukamichi, Eigenfrequencies of vortex state excitations in magnetic submicron-size disks, J. of Appl. Phys., 91(2002), 8037.

[8] V.A.Orlov, G.S.Patrin, I.N.Orlova, Interaction of a Magnetic Vortex with Magnetic Anisotropy Nonuniformity, JETP, 131(2020), no. 4, 589-599.

DOI: $10.1134 /$ S1063776120090071

[9] A.Thiele, Phys. Rev. Lett., 30(1973), 230.

[10] F.G.Mertens, H.J.Schnitzer, A.R.Bishop, Phys. Rev. B, 56(1997), 5, 2510-2520.

[11] M.Wolf, U.K.Robler, R.Schafer, Journal of Magnetism and Magnetic Materials, 314(2007), $105-115$.

[12] Z.Li, D.Dong, D.Liu, J.Liu, D.Liu, X.Li, Phys.Chem.Chem.Phys., 18(2016), 28254.

DOI: $10.1039 / \mathrm{C} 6 \mathrm{CP} 04583 \mathrm{~A}$

[13] V. L. Krutyanskiy, I. A. Kolmychek, B. A. Gribkov, E. A. Karashtin, E. V. Skorohodov, T.V.Murzina, Phys. Rev. B, 88(2013), 094424. DOI: 10.1103/PhysRevB.88.094424

[14] J.P.Chen, Z.Q.Wang, J.J.Gong, M.H.Qin, M.Zeng, X.S.Gao, J.-M.Liu, Journal of Appl. Phys., 113, 054312 (2013). DOI: 10.1063/1.4790483

[15] V.A.Orlov, R.Yu.Rudenko, A.V.Kobyakov, A.V.Lukyanenko P. D. Kim, V. S. Prokopenko, and I. N. Orlova, Magnetization Dynamics in Two-Dimensional Arrays of Square Microelements, JETP, 126(2018), no. 4, 523-534. DOI: 10.1134/S1063776118040118

[16] S.-B.Choe, Y.Acremann, A.Scholl, A.Bauer, A.Doran, J.Stohr, H.A.Padmore, Science, $\mathbf{3 0 4}(2004), 420$.

[17] H.H.Langner, T.Kamionka, M.Martens, M.Weigand, C.F.Adolff, U. Merkt, G. Meier, Phys. Rev. B, 85(2012), 174436. DOI: 10.1103/PhysRevB.85.174436

[18] A.Drews, B.Krüger, G.Selke, T.Kamionka, A Vogel, M.Martens, U.Merkt, D.Möller, G.Meier, Phys. Rev. B, 85(2012), 144417.

[19] J.Kim, S.-B.Choe, J.Magn. 12(2007), no. 3, 113.

[20] P.D.Kim, V.A.Orlov, V.S.Prokopenko et al., On the Low-Frequency Resonance of Magnetic Vortices in Micro and Nanodots, Physics of the Solid State, 57(2015), no. 1, 30-37.

DOI: $10.1134 / \mathrm{S} 1063783415010151$ 
[21] V.A.Orlov, V.S.Prokopenko, R.Yu.Rudenko, I.N.Orlova, Effect of Mechanical Stress on Structure of Magnetization of Three-Layer Nanosized Disks, Physics of Metals and Metallography, 121(2020), no. 11, 1039-1044. DOI: 10.1134/S0031918X20100075

[22] S.S.Zamai, B.S.Prokopenko, V.Ya.Prints, V.A.Seleznev, T.N.Zamai, A.S.Zamai, P.L.Kim, Patent na poleznuyu model, RU 167739 U1, 10.01.2017. Zyavka no. 2015151687 ot 02.12.2015 (in Russian).

\title{
Особенности резонансного поведения намагниченности в массивах треугольных и квадратных наноточек
}

Виталий А. Орлов

Сибирский федеральный университет Красноярск, Российская Федерация Институт физики им. Л. В. Киренского СО РАН Федеральный исследовательский центр КНЦ СО РАН Красноярск, Российская Федерация

Роман Ю. Руденко

Сибирский федеральный университет Красноярск, Российская Федерация Владимир С. Прокопенко Ирина Н. Орлова Красноярский государственный педагогический университет им. В. П. Астафьева Красноярск, Российская Федерация

\begin{abstract}
Аннотация. Теоретически исследуются коллективные моды гиротропного движения ядра магнитного вихря в упорядоченных массивах ферромагнитных пленочных наноточек треугольной и квадратной форм. Получены дисперсионные соотношения. Учитывается диполь-дипольное взаимодействие магнитных моментов ядер магнитных вихрей элементов в приближении малого смещения от положения равновесия. Показано, что эффективная жесткость магнитной подсистемы в треугольных элементах заметно больше, чем в квадратных при одинаковых линейных размерах. Обсуждается перспектива использования пленочных нанодисков-многоугольников в качестве "наноскальпелей" для неинвазивной клеточной хирургии опухолей.
\end{abstract}

Ключевые слова: дифференциальные уравнения, задача Коши, расщепление, устойчивость, сходимость. 